DOI: 10.12957/demetra.2018.30969

\title{
Nutricionistas na atenção primária no município de Santos: atuação e gestão da atenção nutricional
}

\author{
Dietitians in primary health care in the municipality of Santos, São Paulo state: performance \\ and management of nutritional care
}

Natália Spina

Paula Andrea Martins',2

Gabriela Milhassi Vedovato ${ }^{3}$

Anna Sylvia de Campos Laporte ${ }^{3}$

Lia Thieme Oikawa Zangirolani ${ }^{4}$

Maria Angélica Tavares de Medeiros ${ }^{4}$

1 Universidade Federal de São Paulo, Programa de Pós-Graduação em Nutrição. São Paulo, SP, Brasil.

2 Universidade Federal de São Paulo, Instituto Saúde e Sociedade, Departamento de Ciências do Movimento. Santos, SP, Brasil.

${ }^{3}$ Universidade Federal de São Paulo, Instituto Saúde e Sociedade. Santos, SP, Brasil.

${ }^{4}$ Universidade Federal de São Paulo, Instituto Saúde e Sociedade, Departamento de Políticas Públicas e Saúde Coletiva. Santos, SP, Brasil.

Financiamento: Conselho Nacional de Desenvolvimento Científico e Tecnológico (CNPq - processo 486017/2011-7).

Correspondência /Correspondence Maria Angélica Tavares de Medeiros E-mail: angelicamedeiros@gmail.com

\section{Resumo}

Este artigo tem por objetivo analisar a inserção de nutricionistas na Atenção Primária à Saúde no município de Santos, SP, identificando suas práticas na gestão do cuidado nutricional na rede de atenção à saúde. Trata-se de estudo qualitativo, de caráter descritivo, utilizando entrevistas e análise de conteúdo. Realizaram-se entrevistas semiestruturadas com o universo de nutricionistas (três), que dividiam sua atuação nos 28 serviços. As entrevistadas relataram número insuficiente de profissionais, dificultando o trabalho em equipe e a qualidade das ações de alimentação e nutrição. Avaliaram desfavoravelmente seu processo de trabalho, por impossibilitar a atuação em todas as frentes, como a atenção materno-infantil ou ao excesso de peso, e a criação de vínculo com os usuários e com as equipes dos serviços. Embora predominassem atividades em grupo, a maior parte ocorria em salas de espera, sem planejamento prévio, e era de natureza disciplinar. Revelou-se a premência de superar obstáculos, como a carga horária reduzida de trabalho para atuar em cada serviço e a limitada infraestrutura para ações de promoção da saúde e da alimentação adequada e saudável. Conclui-se que o município não possui um número adequado de nutricionistas na Atenção Primária à Saúde, especialmente em áreas de maior vulnerabilidade social, prejudicando os processos de trabalho e a efetivação da integralidade da atenção à saúde.

Palavras-chave: Nutricionistas. Atenção Primária à Saúde. Política Nacional de Alimentação e Nutrição. Sistema Único de Saúde. Prática Profissional. 


\section{Abstract}

This article aims to analyze the insertion of nutritionists in Primary Health Care in the city of Santos, São Paulo state, Brazil, identifying their practices in the management of nutritional care. A qualitative, descriptive study was carried out using interviews and content analysis. Semi-structured interviews were conducted with the universe of nutritionists (three), who divided their performance into 28 services. The interviewees reported insufficient numbers of professionals, making it difficult to work in teams and to assure the quality of food and nutrition actions. They evaluated their work process unfavorably, which makes it hard to act in different fronts, such as maternal and child care or overweight, as well as to build a link with users and professionals. Although group activities predominated, most of them occurred in waiting rooms, without prior planning and were guided by a disciplinary approach. It was found an urgent need to overcome obstacles such as the reduced workload to act in each service and the limited infrastructure for health promotion and adequate and healthy food actions. In conclusion, the municipality does not have an adequate number of nutritionists in Primary Health Care, especially in areas of greater social vulnerability, which undermines work processes and the effectiveness of comprehensive health care.

Keywords: Nutritionists. Primary Health Care. Nutrition Policy. Unified Health System. Professional Practice.

\section{Introdução}

A Atenção Primária à Saúde (APS) compreende o conjunto de ações de saúde organizadas de forma regionalizada e próxima aos indivíduos, às famílias e às comunidades, considerando singularidades e inserção sociocultural, para desenvolver uma atenção integral e humanizada.

Este ponto de atenção é efetivado por meio de práticas de cuidado e gestão de ações voltadas às necessidades da população das áreas de abrangência das Unidades Básicas de Saúde (UBS) tradicionais e das Unidades Saúde da Família (USF), nas quais opera a Estratégia Saúde da Família (ESF). ${ }^{1}$ Nas USF, o contato com os usuários se torna mais próximo, pois as equipes de saúde se deslocam aos territórios em que vivem indivíduos e familiares. Os profissionais inseridos na rede de APS, entre eles os nutricionistas, encontram inúmeros desafios para atuar nesses dois modelos assistenciais. ${ }^{2-6}$ 
Diante da grande influência da alimentação sobre o processo saúde/doença/cuidado, destaca-se a importância de ações, no âmbito individual e coletivo, de Alimentação e Nutrição no contexto da APS. ${ }^{7}$ Essas ações, segundo a Política Nacional de Alimentação e Nutrição (PNAN) ${ }^{8}$, operam em diferentes níveis de intervenção para promoção da saúde, prevenção, diagnóstico, assistência, tratamento e reabilitação de doenças, além do compromisso com a Segurança Alimentar e Nutricional e a exigibilidade dos direitos humanos à saúde e à alimentação adequada e saudável. ${ }^{7}$

Nessa perspectiva, nutricionistas e toda a equipe de saúde desempenham papel central em diferentes frentes de ação, tais como vigilância alimentar e nutricional, atenção ao pré-natal e puerpério, saúde da criança, prevenção e controle de doenças crônicas não transmissíveis (DCNT) e saúde do idoso. Os processos de trabalho e as possibilidades de atuação interdisciplinar para o cuidado integral nas diferentes fases da vida, além da articulação de redes, constituem os aspectos mais frágeis e que necessitam ser enfrentados pela equipe de saúde para atender aos preceitos de integralidade na atenção à saúde no Brasil..2,10,11

O nutricionista, inserido na equipe multiprofissional na Atenção Primária à Saúde, possui competências e atribuições para atuar em diferentes frentes de ação, de caráter universal e específico.,12 A formação deste profissional é a base para o direcionamento e o desempenho profissional na área de Saúde Coletiva. ${ }^{13-15}$ Experiências de formação acadêmica do nutricionista, pautadas no trabalho interdisciplinar e no reconhecimento do território, para o desenvolvimento de olhar e escuta sensíveis aos problemas de saúde, vêm-se mostrando efetivas para potencializar a atenção integral à saúde..$^{16,17}$

O desenvolvimento de competências e a construção de identidade profissional do nutricionista são aspectos problematizados na literatura, ${ }^{15,18}$ apontando para a necessidade de fortalecer a formação voltada à APS. ${ }^{13,19}$ No tocante à atuação nessa área, estudos apontam dificuldades relativas à inserção ainda insuficiente deste profissional para responder às demandas de atenção integral à saúde.,3,10,11

Desta forma, entender os desafios para a prática profissional na rede de Atenção Primária à Saúde, na perspectiva do profissional nutricionista, pode nortear ações mais efetivas e consoantes entre a formulação de políticas públicas e as práticas locais, além de poder guiar processos relacionados à formação acadêmico-profissional. Este artigo tem como propósito analisar a inserção de nutricionistas na APS no município de Santos, SP, buscando identificar suas práticas nas diferentes frentes de ação que compõem a gestão do cuidado nutricional na rede. 


\section{Procedimentos metodológicos}

Trata-se de estudo com abordagem qualitativa, de caráter descritivo, no qual se investigou o universo de nutricionistas inseridos na rede de Atenção Primária à Saúde de Santos, SP. ${ }^{a}$

O município de Santos possui uma população de 419.400 habitantes e se divide em duas áreas geográficas - insular e continental -, sendo que esta última tem $70 \%$ do território ocupado por área de preservação ambiental. A área insular, com 99\% da população, é dividida em quatro regiões administrativas de saúde: Centro, Morros, Orla/Intermediária e Zona Noroeste. A região da Orla possui melhor condição socioeconômica, enquanto as outras três apresentam maior desigualdade social e população em situações de maior vulnerabilidade. ${ }^{20}$ Neste estudo, a área continental foi excluída, devido às dificuldades de acesso e à baixa densidade populacional. Na área insular, há um total de 28 Unidades de Atenção Primária distribuídas pelas quatro regiões, das quais 19 são UBS e nove USF.

Foram realizadas entrevistas semiestruturadas com todos os nutricionistas da Atenção Primária à Saúde, utilizando um roteiro aprimorado por estudo-piloto, para investigar a inserção desses profissionais na rede e identificar aspectos relativos à atuação e à gestão do cuidado nutricional. O instrumento compreendia os seguintes domínios: dados gerais relativos ao perfil profissional; adequação/suficiência de recursos; processo de trabalho; ações de alimentação e nutrição nas diferentes fases do ciclo da vida.

Processo de trabalho, aqui, é entendido como a organização de práticas profissionais que tenham o usuário como centro, em detrimento de protocolos preestabelecidos, contribuindo, assim, para a integralidade do cuidado em saúde. ${ }^{1}$

As entrevistas foram realizadas no ano de 2013, no próprio local de trabalho (Unidades de Saúde da Família e/ou Unidades Básicas de Saúde), com duração de cerca de 60 minutos cada. Os nutricionistas foram entrevistados pela pesquisadora responsável, juntamente com uma pesquisadora assistente, ambas capacitadas para mediar e observar o processo de entrevistas. Paralelamente, eram realizadas supervisões de campo, para conferência dos dados coletados e verificação das dificuldades encontradas no momento da entrevista.

As entrevistas foram conduzidas de maneira a estimular que os participantes falassem livremente sobre tópicos pertinentes às dimensões relativas às práticas, nas diferentes ações de alimentação e nutrição, e aos processos de trabalho, adequação e suficiência de recursos locais. Esta abordagem exploratória favorece a compreensão dos significados das ações e da realidade vivenciada pelos participantes.

a O estudo constitui parte da pesquisa "A integralidade da atenção e as ações de nutrição na Atenção Básica de Santos" (CNPq 486017/2011-7). ${ }^{17}$ 
A sistematização e a análise de conteúdo dos dados oriundos das entrevistas foram guiadas pelas recomendações metodológicas de Bardin. ${ }^{21}$ Primeiramente, foi feita a organização dos dados (respostas das questões e notas de campo das observações), seguida por uma leitura flutuante preliminar do material empírico, para estabelecer conexão entre as questões de pesquisa e a elaboração de uma estrutura analítica axial alinhada às dimensões do roteiro da entrevista.

Posteriormente, iniciou-se o processo analítico de codificação, utilizando o critério semântico, no qual os discursos foram agregados de forma objetiva, precisa e rigorosa, gerando as unidades de registro. A essas unidades de registro foram atribuídos temas identificados pelas pesquisadoras, a partir do conteúdo do discurso dos participantes. Em um último momento, foi feito um refinamento dos códigos, por meio do agrupamento em categorias temáticas. Os resultados foram apresentados por dimensões organizadas com trechos de citações dos participantes, para ilustrar cada uma das categorias temáticas identificadas.

A pesquisa foi autorizada pela Secretaria Municipal da Saúde de Santos e aprovada pelo Comitê de Ética em Pesquisa da Universidade de origem, sob o parecer $n^{\circ}$. 32900/2012. Os voluntários assinaram o Termo de Consentimento Livre e Esclarecido para a participação no estudo.

\section{Resultados e Discussão}

Foram entrevistadas as três nutricionistas, mulheres e servidoras públicas da Secretaria Municipal de Saúde, que atuavam na rede de Atenção Primária à Saúde de Santos. Essas profissionais se graduaram há, respectivamente, 28 (N1), 10 (N2) e 08 (N3) anos. Todas trabalhavam na rede há mais de três anos. Quanto à qualificação profissional, duas nutricionistas cursavam pós-graduação no momento da entrevista, sendo uma delas em mestrado profissional (stricto sensu) e a outra em especialização (lato sensu).

Do total de 28 unidades de saúde da área insular de Santos, 19 (68\%) contavam com a atuação direta das entrevistadas, sendo 13 UBS e seis USF. Cada nutricionista relatou uma jornada de trabalho de 40 horas semanais e sua inserção nessas unidades se dava entre um período de quatro horas semanais, em 16 unidades, e dois períodos, totalizando oito horas semanais, em três outras unidades.

O número de nutricionistas atuantes na APS do município está abaixo do preconizado pelo órgão regulamentador da profissão (i.e. um profissional para cada 30 mil habitantes) em todas as regiões. ${ }^{22}$ Nas regiões Centro, que possui seis unidades de saúde, com uma população adscrita de 88.900 habitantes; Morro, com oito unidades de saúde e 62.000 habitantes, e Orla, com seis unidades de saúde e 187.950 habitantes, existe apenas uma nutricionista por região. Segundo o Conselho Federal de Nutricionistas, a recomendação seria de três, duas e seis profissionais, 
respectivamente, por unidade de saúde. ${ }^{22}$ Especificamente na Zona Noroeste, região de grande vulnerabilidade social, ${ }^{20}$ que conta com oito unidades de saúde e uma população adscrita de 71.820 habitantes, não há nutricionista inserido em nenhuma das oito unidades.

Verificou-se que a inserção do nutricionista na APS do município de Santos é insuficiente para atender à população nas áreas de abrangência das Unidades Básicas de Saúde e Unidades de Saúde da Família. Igualmente, estudos na região metropolitana de Campinas-SP ${ }^{23}$ e no município de São Paulo $^{9}$ constataram uma quantidade insuficiente de nutricionistas na Atenção Primária à Saúde, quando comparada à recomendação.

Em estudo realizado no mesmo município do presente estudo, ${ }^{24}$ foram entrevistados gestores de unidades de saúde e evidenciou-se que, para $60 \%$ deles, a insuficiência de nutricionistas é um problema estrutural que compromete a atenção nutricional.

A inadequação da inserção de nutricionistas na rede de Atenção Primária à Saúde exerce impacto negativo sobre o modo de organizar o trabalho, especialmente em termos de caráter e resolutividade das ações desenvolvidas. ${ }^{3,15,23}$ Ainda, a escassez de trabalhadores na área da saúde pode restringir as ações ao serviço de saúde, impossibilitando articulações intersetoriais no território de abrangência e restringindo a prospecção de iniciativas no âmbito político. ${ }^{25}$

A partir da análise das falas das participantes, foi possível identificar estas quatro categorias temáticas: processo de trabalho, construção de vínculo (com as equipes de saúde e com os usuários), caráter das ações desenvolvidas e parceria universidade/serviço.

\section{Processo de trabalho}

As participantes manifestaram insatisfação com seu processo de trabalho, pela impossibilidade de atuar em todas as fases do ciclo da vida (o que uma delas chamou de "linha de cuidado"), levando-as a privilegiar frentes de atuação de maior afinidade, em detrimento de outras.

Me sinto insatisfeita por não conseguir trabalhar em todas as linhas de cuidado. É preciso descobrir seu espaço, acabo focando na linha materno-infantil, que é a com que mais me identifico (N1).

Além disso, essa insatisfação se mostrou associada à dificuldade em dar seguimento às ações de atenção nutricional, devido à restrição do tempo de trabalho dedicado a cada unidade.

[...] é desgastante e ao mesmo tempo decepcionante, porque não conseguimos acompanhar e dar continuidade ao trabalho realizado, devido à correria e ao pouco tempo nas unidades (N2).

Pádua \& Boog ${ }^{23}$ observaram que a insatisfação de nutricionistas inseridas na rede de APS da região metropolitana de Campinas-SP esteve mais relacionada à baixa remuneração, ao excesso 
de demanda de trabalho e à necessidade de demonstrar competência profissional frente aos desafios no trabalho.

A precariedade da infraestrutura das unidades e as limitações de recursos físicos, materiais e de profissionais foram apontadas, pelas entrevistadas, como condições diretamente relacionadas à insatisfação no trabalho que precisam ser superadas. Isto porque inviabilizam e reduzem o escopo de atuação dos nutricionistas e, possivelmente, a qualidade das ações de Alimentação e Nutrição desenvolvidas na rede.

Indagadas sobre o número de profissionais de saúde em cada serviço, todas as nutricionistas avaliaram como insuficiente para atuar nas distintas demandas, sejam elas de caráter individual ou coletivo. Igualmente identificaram limitações de espaço físico para o manejo de medidas antropométricas, assim como a insuficiência de equipamentos, em alguns dos locais em que atuavam.

Acho que poderia melhorar a área física e o número de profissionais envolvidos. (N3)

A gestão do cuidado nutricional pode ser bastante fragilizada por entraves organizacionais, estruturais e funcionais que interferem na dinâmica de atuação profissional na Atenção Primária à Saúde. ${ }^{4}$ A escassez de nutricionistas e de outros profissionais da saúde - que podem ser chamados de gestores do cuidado - diminui a fluidez das estratégias coletivas e individualizadas, na perspectiva da integralidade da atenção à saúde. ${ }^{26}$

\section{Construção de vínculo (com as equipes de saúde e com os usuários)}

As participantes relataram que um número adequado de profissionais e maior comunicação entre eles, viabilizada, por exemplo, por reuniões de equipe, poderiam fortalecer as relações e facilitar a articulação de estratégias de vínculo, tanto com os profissionais quanto com a população usuária dos serviços, e o encaminhamento de demandas dos usuários nas unidades de saúde.

Em algumas unidades, é necessária a realização de reuniões em equipe, favorecendo a aproximação dos profissionais e o estabelecimento do vínculo com a população (N1).

[...] Nas UBS, o reduzido número de funcionários e a grande demanda impedem o relacionamento mais próximo com o usuário (N2).

A participação dos profissionais nas discussões de caso é essencial para que entendam a realidade dos usuários (N3)

A gestão compartilhada, propiciada pelas reuniões de equipe, é capaz de despertar o espírito de responsabilidade coletiva na atenção à saúde, o que constitui mecanismo vital para a continuidade 
das práticas específicas de cada profissional, ampliando a chance de efetividade das ações interdisciplinares na rede de atenção à saúde. ${ }^{4,25}$ Um relato de experiência, de estágios curriculares interdisciplinares em Nutrição e Psicologia, evidenciou o importante papel das reuniões de equipe para discussão de casos, na construção do projeto terapêutico singular, na elaboração de protocolos de atenção e de planos estratégicos para ações de educação em saúde no município de Santos. ${ }^{27}$

Neste estudo, as nutricionistas entrevistadas referiram diferenças nos formatos de ações entre ambas as modalidades assistenciais. Segundo elas, a dinâmica relativa ao arranjo e ao modelo de atenção nas Unidades de Saúde da Família favorece o acolhimento das demandas reais da população nos territórios, além da construção de vínculo. A atenção domiciliar, em especial, é vista como uma abordagem que permite ainda maior proximidade entre a equipe de saúde e a população.

Geralmente, nas USF, os funcionários conhecem os usuários e os chamam pelo nome [...] é diferente porque o contato com os munícipes é mais frequente (N2).

Desde a chegada do usuário na USF, o vínculo e o acolhimento estão presentes [...] as visitas domiciliares também fortalecem o vínculo. (N1)

Na reorganização do modelo assistencial, a partir da Estratégia Saúde da Família, aproximar a realidade dos indivíduos, famílias e comunidade da equipe de saúde configura-se como essencial para garantir a integralidade e a humanização do cuidado. ${ }^{28,29}$ Neste sentido, o cenário domiciliar deve ser concebido de forma ampliada pelos profissionais, ou seja, para além dos aspectos físicos do ambiente e dos comportamentais dos indivíduos. Isso implica considerar a história de vida, o contexto em que se inserem e o repertório cultural dos mesmos. ${ }^{4,33} \mathrm{O}$ trabalho multiprofissional e interdisciplinar, centrado na família, assume caráter estratégico para dimensionar as complexidades e trabalhar questões relativas aos elementos e sujeitos que interagem entre si, e que influenciam o processo de saúde e doença. ${ }^{30,31}$

A partir da representação social de profissionais de equipes de saúde da família, no município de São Carlos-SP, Camossa et al. ${ }^{2}$ observaram o papel significativo do nutricionista dos Núcleos de Apoio à Saúde da Família (NASF) nas visitas domiciliares, favorecendo práticas de intervenção nutricional mais adequadas à realidade da população, além de estreitamento do vínculo com a população e maior adesão ao tratamento.

A escuta comprometida com o desenvolvimento da autonomia dos munícipes e a ampliação da resolutividade das intervenções em saúde apresentam relação direta com o estabelecimento de vínculo entre profissionais de saúde e população, por estarem associadas à construção de relações de confiança, humanizadas e pautadas na compreensão das necessidades reais do indivíduo assistido. ${ }^{32,33}$ 
Segundo as nutricionistas entrevistadas, a maior interação interprofissional, conferida pelo modelo de atenção nas Unidades de Saúde da Família, gera um trabalho mais integrado e satisfatório. Também se observou, nos discursos, a necessidade de ressignificação e reformulação do trabalho em equipe nas Unidades Básicas de Saúde.

[...] Nas USF, o fato de podermos realizar trabalhos em equipe multiprofissional é muito positivo. Em unidades em que este trabalho não acontece, o papel do profissional fica meio perdido, você tem que procurar seu espaço, é um trabalho mais isolado (N1).

As USF têm um modelo de trabalho que propicia este envolvimento (N3).

Desta forma, ressalta-se o papel da ESF de pautar suas ações no trabalho interdisciplinar. ${ }^{4} \mathrm{O}$ modelo assistencial das USF desloca o foco da atuação médico-centrada para o trabalho em equipe multiprofissional, permitindo o compartilhamento e a integração de conhecimentos, habilidades e competências, para fomentar o cuidado integral. Entretanto, ainda se identificam dissonâncias entre os fundamentos deste modelo assistencial e a prática dos serviços. ${ }^{5,9,10,13,26}$

Em algumas situações, o nutricionista enfrenta dificuldades para uma inserção mais efetiva na equipe de saúde da família, por desconhecimento de outros profissionais quanto à sua atuação específica, ${ }^{11}$ ou por ser identificado como um profissional coadjuvante do médico, o que restringe sua atuação e autonomia profissional na atenção à saúde. ${ }^{2}$ Em contrapartida, o trabalho em saúde demanda a formação de vínculo entre os próprios profissionais, como requisito para o trabalho em equipe e o compartilhamento de saberes e práticas que configuram uma atuação interdisciplinar. ${ }^{11}$

\section{Caráter das ações desenvolvidas}

Em relação aos tipos de ação e às abordagens desenvolvidas pelas nutricionistas da Atenção Primária de Santos, verificou-se o predomínio de atividades em grupo na rotina de trabalho, pelo potencial de abarcar maior número de munícipes, comparativamente ao atendimento individual. Contudo, a maior parte dos grupos educativos era feita em salas de espera, carecendo de planejamento prévio e de continuidade.

Por essas características, tais grupos eram limitados em seu potencial de promover autonomia e empoderamento dos sujeitos, ${ }^{34}$ sendo mais um arranjo que as profissionais encontravam para responder à grande demanda por atenção nutricional, em relação a suas cargas de trabalho em cada local. Ademais, a natureza das atividades em grupo era, usualmente, disciplinar. Em geral, os grupos abordavam temáticas como incentivo ao aleitamento materno ou promoção da alimentação saudável nas DCNTs. 
Faço mais hiperdia [grupo de sala de espera], devido ao grande volume de pacientes... alguns grupos de gestantes ou insulinodependentes.(N2). [Grifos nossos].

Novamente aparece aqui a precariedade das condições de trabalho impactando no planejamento de ações de educação alimentar e nutricional, conforme ilustram as falas abaixo:

Pelo número de unidades [em que trabalha], fica difícil organizar, construir, porque já chega para executar (N3) [Grifos nossos].

[...] no geral, o número de unidades deveria ser reduzido. (N1)

Similarmente, Cervato-Mancuso et al. ${ }^{3}$ identificaram predominância de atividades coletivas, como grupos educativos e atendimentos compartilhados, focados, respectivamente, em promoção da saúde e prevenção de doenças, como sendo as mais realizadas por nutricionistas do NASF do município de São Paulo.

Destaca-se a relevância da atuação do nutricionista no campo de educação em Saúde Coletiva, para a concretização de iniciativas em Segurança Alimentar e Nutricional e Promoção da Saúde. ${ }^{16}$ Nesta perspectiva, além de suas competências e atribuições específicas no planejamento, na execução e na avaliação de atividades de educação alimentar e nutricional, há necessidade de desenvolver atividades terapêuticas e educativas interdisciplinares, para tratar da complexidade de fenômenos relacionados às práticas alimentares e de saúde. ${ }^{12,18}$

Frente ao fato de que a maioria dos grupos, conduzidos pelas nutricionistas entrevistadas, acontecia em salas de espera para o atendimento clínico, é necessária precaução para que as abordagens adotadas não assumam caráter prescritivo e meramente informativo.

No Brasil, ainda há uma lacuna entre os referenciais teóricos e as práticas locais de educação alimentar e nutricional, dado que muitas abordagens não são pautadas na dialogicidade e na problematização da realidade dos indivíduos, para o desenvolvimento de reflexão e de autonomia. São, portanto, pouco efetivas para a promoção da saúde e a prevenção de doenças. ${ }^{2,5,35}$

Importante ressaltar que o planejamento constitui um processo de trabalho preliminar, necessário para aumentar a chance de sucesso das ações desenvolvidas pelo nutricionista na Atenção Primária à Saúde. ${ }^{34}$ A elaboração de planos de ação que incluam etapas de diagnóstico nutricional e educativo, além de métodos de avaliação processual e de impacto, é uma prática primordial para proporcionar adequação, efetividade e aperfeiçoamento das estratégias adotadas. ${ }^{36}$

A ausência de planejamento e de organização de um fluxo terapêutico, com a desarticulação entre estratégias coletivas e individualizadas, impede a efetivação da integralidade da atenção em todas as fases do ciclo da vida. ${ }^{3,10} \mathrm{Na}$ APS em exame, o atendimento nutricional individual era 
realizado a partir de encaminhamento médico, segundo as nutricionistas. Uma das entrevistadas, contudo, relatou desenvolver atendimento individual como parte principal da rotina em duas unidades de saúde; a maioria dos encaminhamentos se referia a demandas por orientação nutricional para tratamento de DCNTs.

Verificou-se ainda que, na ausência de nutricionistas, outros profissionais de saúde da unidade realizavam ações de Alimentação e Nutrição. Por exemplo, orientações sobre alimentação complementar eram conduzidas, preponderantemente, pelos médicos, sendo que em apenas cinco unidades de Santos, essa atividade era exercida por nutricionistas. A fala abaixo sintetiza tal constatação:

[...] auxiliares de enfermagem abordam alimentação durante aferição de pressão arterial; médicos abordam nas consultas e pediatras falam de alimentação complementar. (N1)

Este achado corrobora os de outros estudos que mostraram que médicos e enfermeiros realizam, comumente, orientações nutricionais em serviços de Atenção Primária à Saúde, ${ }^{2,23}$ evidenciando um modelo prescritivo, de imposição de normas alimentares e de repasse de orientações generalizadas e não adequadas às singularidades individuais e à realidade local.

Esse modelo de atenção nutricional, reproduzido por profissionais de saúde, pode estar associado a representações sociais que reduzem o papel dos nutricionistas ao de simplesmente "passar dietas". E ainda, o trabalho do nutricionista, em algumas situações, pode ser interpretado como auxiliar ao atendimento médico, o que fere princípios da interdisciplinaridade e da integralidade do cuidado. ${ }^{9}$ Nesse sentido, são necessárias ações de capacitação da equipe de saúde, desenhadas em formato de apoio matricial, para sensibilizar sobre a importância das atribuições do nutricionista e das ações interdisciplinares na área de Alimentação e Nutrição. ${ }^{2,24}$

A despeito destes resultados, é necessário salientar que condutas prescritivas e normativas na atenção nutricional também são verificadas entre nutricionistas e que, por outro lado, nem todos os outros profissionais de saúde se apoiam nessa perspectiva. Abordagens profissionais mais abrangentes passam, entre outras questões, pela formação para o trabalho em saúde e pela concepção sobre o processo saúde-doença-cuidado adotada pelo profissional em questão. 16,17,27,37

Outra questão que merece reflexão diz respeito ao fato de nutricionistas se restringirem ao papel de "passar dietas", ou serem considerados como auxiliares de médicos. Neste caso, é relevante apontar que, no universo da formação de nutricionistas, identificam-se profissionais que encontram significado e sentido em se colocar exatamente neste lugar. Isso remete à tendência contemporânea de medicalização do ato de comer, dada pela supervalorização de nutrientes, em detrimento da comida de verdade e desrespeitando a carga cultural trazida pelos indivíduos e grupos populacionais quanto à alimentação. Para tanto, reivindicam-se profissionais especialistas, 
ocupados em prescrever condutas nutricionais, geralmente distantes da cultura alimentar da população, tomando este ou aquele nutriente como princípio da saúde, sob a ótica biomédica. ${ }^{38}$

Nas unidades estudadas no município de Santos, a avaliação antropométrica foi relatada como uma atividade frequentemente realizada por enfermeiros e técnicos de enfermagem, como parte da rotina de atendimentos. Entretanto, a insuficiência de nutricionistas foi percebida como uma limitação para o desenvolvimento de ações de capacitação direcionadas aos profissionais da rede de APS, não nutricionistas, para lidar com as dificuldades vivenciadas pela falta de domínio teórico e prático para especificidades nesta área.

Os outros profissionais da equipe têm dificuldade teórica em relação às questões de nutrição, pois não têm conhecimento específico [...] eles sentem a ausência do nutricionista nas unidades (N1).

As entrevistadas avaliaram como parcialmente efetivas as ações desenvolvidas por elas nas diferentes fases da vida. Atividades educativas realizadas no Programa Hiperdia - Sistema de Gestão Clínica de Hipertensão Arterial e Diabetes Mellitus da Atenção Básica (que ocorrem em $15 \mathrm{UBS} / \mathrm{USF}$ ) foram as mais frequentemente realizadas pelas nutricionistas.

Todavia, na visão dessas profissionais, a atenção voltada aos munícipes com diabetes e hipertensão arterial enfoca, primariamente, o controle e o tratamento dessas doenças crônicas, nos quais as atividades em geral estão atreladas à aferição de glicemia capilar, pressão arterial e à entrega de insumos para automonitoramento e tratamento. Além disso, as profissionais relataram haver pouca disponibilidade de tempo para desenvolver ações educativas de promoção da saúde e prevenção de doenças, na perspectiva da família e da comunidade, sugerindo fragilidades na efetividade dessas ações.

Sobre a atenção nutricional no pré-natal e no puerpério, as nutricionistas avaliaram as atividades desenvolvidas como parcialmente ineficazes na maioria das unidades, identificando como obstáculos centrais: número insuficiente de nutricionistas, baixo reconhecimento do papel desse profissional pela equipe e grande demanda nas áreas de abrangência.

[...] falta de tempo da nutricionista e falta de conhecimento da importância disso por parte dos profissionais. (N1)

[...] precisaria de tempo maior nas unidades e de envolvimento de outros profissionais. (N3).

Estudo prévio, com gestores da Atenção Primária do município em estudo, identificou uma série de inadequações de processo dos serviços para a concretização da atenção nutricional no pré-natal e no puerpério, tais como baixa proporção de acompanhamento do estado nutricional gestacional e de aconselhamento nutricional individualizado no pré-natal. ${ }^{24}$ As autoras verificaram 
que a limitada disponibilidade e a não manutenção de equipamentos de antropometria, além da inexistência de estrutura física para atendimentos individuais, grupos educativos/terapêuticos e reuniões de equipe, constituíram entraves capazes de inviabilizar um fluxo terapêutico adequado na linha de cuidado materno-infantil.

As participantes avaliaram a atenção nutricional na infância e aos idosos como sendo igualmente insatisfatórias nas unidades em que se inseriam. Estes resultados revelam a necessidade de maior planejamento e organização da atenção nutricional, em um cenário carente da atuação do nutricionista, de maneira a abarcar as diversas frentes de atuação e desenvolver ações que potencializem os recursos físicos disponíveis e a ação dos profissionais.

\section{Parceria universidade/serviço}

Pelas próprias limitações de tempo das nutricionistas para o desenvolvimento de ações intersetoriais, a parceria universidade/serviço foi descrita como a única ação intersetorial realizada pelas entrevistadas. Das 19 Unidades em que há atuação das nutricionistas, nove têm parcerias com universidades (uma pública e duas privadas) e contam com a inserção de estudantes de graduação e pós-graduação.

Entre as atividades acadêmicas desenvolvidas nos serviços pela universidade pública, destacamse projetos de extensão universitária (uma unidade); práticas curriculares da graduação focadas no trabalho em saúde, voltadas à formação interdisciplinar combinando estudantes dos diversos cursos nos três primeiros anos da graduação; e estágios curriculares no quarto ano dos cursos de graduação (nove unidades). Nesses períodos, trabalham-se questões transversais e comuns a todos os profissionais de saúde, com atividades que ocorrem na Rede de Atenção Primária à Saúde. ${ }^{37}$

As nutricionistas entrevistadas, as quais supervisionam as atividades acadêmicas em campo, relataram que os estudantes têm grande envolvimento com as ações por elas desenvolvidas e que a parceria serviço-universidade é positiva.

Todos ganham com esta parceria, a população, os alunos e a Unidade. As atividades desenvolvidas são ótimas. (N1)

Por outro lado, identificaram dificuldades nesta relação:

Os estágios poderiam ser mais estruturados, a nutricionista não consegue dar muita atenção, pois não está todos os dias na unidade. (N1) 
Há registros de experiências significativas, em termos de formação acadêmica, profissional e pessoal, que emergem das interações e do encontro com diferentes realidades de grupos populacionais que vivem em áreas de maior vulnerabilidade social. ${ }^{27,37}$ Desta forma, o diálogo entre a formação inicial e a realidade dos serviços e territórios é imprescindível para despertar uma reflexão crítica do estudante, futuro nutricionista, sobre seu papel e inserção na rede de Atenção Primária à Saúde, uma vez que a atuação de nutricionistas no NASF vem mostrando alguns descompassos e fragilidades em relação à formação acadêmica e profissional., 55

A despeito da importância da parceria universidade e serviço de saúde, tanto para a formação em graduação quanto para a qualificação das equipes, não se pode deixar de mencionar os limites que tal relação pode encerrar. Notadamente, é preciso atentar para que esta parceria não camufle a necessidade de maior inserção de nutricionistas nos serviços, no que tange tanto à necessidade de contratação de maior número destes profissionais, quanto à demanda de qualificação e ampliação das ações por eles realizadas, com vistas à atenção integral à saúde. A esse respeito, a literatura mostra que, apesar dos avanços, a inserção destes profissionais segue insuficiente, frente às demandas da APS. ${ }^{2,3,19}$

\section{Consideraçóes finais}

Neste estudo, analisaram-se as práticas de nutricionistas na Atenção Primária de Santos, o que permitiu identificar barreiras para o desempenho de suas atribuições.

Duas limitações do estudo devem ser apontadas. A primeira diz respeito ao caráter contextoespecífico, que não permite a generalização dos achados para outras estruturas de organização de redes de Atenção Primária à Saúde. A segunda limitação relaciona-se ao fato de que técnicas complementares de pesquisa (por exemplo, observação participante e narrativa) poderiam ter sido empregadas, para proporcionar uma triangulação analítica e o aprofundamento de suas percepções e práticas.

Verificou-se que o município de Santos, importante centro urbano na região metropolitana da Baixada Santista, não possui um número adequado de nutricionistas inseridos na Atenção Primária à Saúde, especialmente em áreas de maior vulnerabilidade social. O número insuficiente de profissionais prejudica a qualidade dos processos de trabalho nas unidades de saúde e a efetivação da integralidade da atenção na gestão do cuidado.

Neste cenário, a restrição de tempo dedicado a cada unidade foi apontada como um entrave, pois dificulta o trabalho nas diversas frentes de ação e a construção de um fluxo terapêutico para o cuidado nutricional nas diferentes fases do ciclo da vida. Muitas ações assumem caráter essencialmente assistencial, ainda que realizadas em grupos e focadas no tratamento e na reabilitação de doenças. Desta forma, falta tempo para o planejamento com enfoque coletivo na prevenção 
de doenças, na promoção da saúde, assim como para o desenvolvimento de ações nos territórios em que atuam, envolvendo outros setores, além da saúde. Este contexto mostrou-se associado à insatisfação em relação ao trabalho desempenhado pelas profissionais na rede de APS de Santos.

Conclui-se, a partir das falas das nutricionistas entrevistadas, que tal realidade limita a organização e o desenvolvimento da atenção nutricional na Atenção Primária à Saúde do município.

Por fim, destacam-se como principais contribuições deste estudo a reflexão sobre a importância do nutricionista na Atenção Primária à Saúde e a identificação dos obstáculos enfrentados por esse profissional para a integração, a continuidade e o aperfeiçoamento de suas ações. Portanto, são necessárias ações, em âmbito político-institucional, para a sensibilização de tomadores de decisão no município, de modo a garantir adequação de recursos, materiais, estruturais e de profissionais, que viabilizem o trabalho em equipe e a organização da atenção nutricional no SUS de Santos.

\section{Colaboradores}

Spina N redigiu o manuscrito e contribuiu com a análise, discussão dos resultados e aprovação da versão final do manuscrito; Laporte ASC contribuiu com a concepção do estudo, discussão dos resultados, revisão e aprovação da versão final do manuscrito; Vedovato GM contribuiu com a redação, análise e discussão dos resultados, revisão e aprovação da versão final do manuscrito; Zangirolani LTO e Martins PA contribuíram com a discussão dos resultados, revisão e aprovação da versão final do manuscrito; de Medeiros MAT contribuiu com a concepção, análise e discussão dos resultados e com a revisão e aprovação da versão final do manuscrito.

Conflito de Interesses: As autoras declaram não haver conflito de interesses.

\section{Agradecimentos}

Ao Conselho Nacional de Desenvolvimento Científico e Tecnológico (CNPq), pelo financiamento recebido (CNPq - processo 486017/2011-7).

À Secretaria de Saúde do Município de Santos, pela autorização para o desenvolvimento deste estudo.

\section{Referências}

1. Brasil. Ministério da Saúde. Secretaria de Atenção à Saúde. Departamento de Atenção Básica. PNAB - Política Nacional de Atenção Básica. Brasília: Ministério da Saúde; 2012.

2. Camossa ACA, Telarolli Junior R, Machado MLT. O fazer teórico-prático do nutricionista na estratégia saúde da família: representações sociais dos profissionais das equipes. Rev Nutr. 2012; 25(1):89-106. 
3. Cervato-Mancuso AM, Tonacio LV, Silva ER, Vieira VL. A atuação do nutricionista na Atenção Básica à Saúde em um grande centro urbano. Cien Saúde Coletiva. 2012; 17(12):3289-3300.

4. Cotta RMM, Schott M, Azeredo CM, Franceschini SDCC, Priore SE, Dias G. Organização do trabalho e perfil dos profissionais do Programa Saúde da Família: um desafio na reestruturação da atenção básica em saúde. Epidemiol e Serviços Saúde. 2006; 15(3):7-18.

5. Rodrigues DCM, Bosi MLM. O lugar do nutricionista nos Núcleos de Apoio à Saúde da Família. Rev Nutr. 2014; 27(6):735-746.

6. Vieira VL, Gregório MJ, Cervato-Mancuso AM, Graça APSR. Ações de alimentação e nutrição e sua interface com segurança alimentar e nutricional: uma comparação entre Brasil e Portugal. Saúde Soc. 2013; 22(2):603-607.

7. Brasil. Ministério da Saúde. Secretaria de Atenção à Saúde. Departamento de Atenção Básica. Matriz de ações de alimentação e nutrição na Atenção Básica de Saúde. Brasília: Ministério da Saúde; 2009.

8. Brasil. Ministério da Saúde. Departamento de Atenção Básica. PNAN -Política Nacional de Alimentação e Nutrição. Brasília: Ministério da Saúde; 2012.

9. Loch-Neckel G, Seemann G, Eidt HB, Rabuske MM, Crepaldi MA. Desafios para a ação interdisciplinar na atenção básica: implicações relativas à composição das equipes de saúde da família. Cienc Saúde Coletiva. 2009; 14(Supl. 1):1463-1472.

10. Neves JA, Zangirolani LTO, Medeiros MAT. Evaluation of nutritional care of overweight adults from the perspective of comprehensive health care. Rev Nutr. 2017; 30(4):511-524.

11. Santos IG, Batista NA, Devincenzi MU. Residência Multiprofissional em Saúde da Família: concepção de profissionais de saúde sobre a atuação do nutricionista. Interface - Comun Saúde, Educ. 2015; 19(53):349-360.

12. Brasil. Conselho Federal de Nutricionistas. O papel do nutricionista na Atenção Primária à Saúde. Brasília: CFN; 2008. Disponível em: http://www.cfn.org.br/eficiente/repositorio/Cartilhas/61.pdf

13. Alves CGL, Martinez MR. Lacunas entre a formação do nutricionista e o perfil de competências para atuação no Sistema Único de Saúde (SUS). Interface - Comunicação, Saúde, Educação. 2016; 20(56):159-169.

14. Shrimpton R, Hughes R, Recine E, Mason JB, Sanders D, Marks GC, et al. Nutrition capacity development: a practice framework. Public Health Nutr. 2014; 17(3)682-688.

15. Recine E, Sugai A, Monteiro RA, Rizzolo A, Fagundes A. Saúde coletiva nos cursos de Nutrição: análise de projetos político-pedagógicos e planos de ensino. Rev Nutr. 2014; 27(6):747-760.

16. Cruz PJSC, Melo Neto JF. Educação popular e nutrição social: considerações teóricas sobre um diálogo possível. Interface - Comun Saúde, Educ. 2014; 18(Suppl. 2):1365-1376.

17. Medeiros MAT, Martins PA, Braga-Campos FC, Laporte ASC, Zangiraloni LTO. A integralidade da atenção e as ações de nutrição na Atenção Básica de Saúde. Relatório final de pesquisa. Universal CNPq. Processo n. 486017-2011-7. Santos, SP; 2014. (Mimeo).

18. Medeiros MAT, Amparo-Santos L, Domene SMÁ. Education of dietitian's in Brazil: minimum clock hours of instruction for a bachelor's degree in nutrition. Rev Nutr. 2013; 26(5):583-593. 
19. Vasconcelos IAL, Sousa MF, Santos LMP. Evolução do quantitativo de nutricionistas na Atenção Básica do Brasil: a contribuição dos Núcleos de Apoio à Saúde da Família e da Estratégia Saúde da Família de 2007 a 2013. Rev Nutr. 2015; 28(4):431-450.

20. Instituto Brasileiro de Geografia e Estatística. Cidades - Santos. Censo 2010 [Internet]. Disponível em: https://cidades.ibge.gov.br/brasil/sp/santos/panorama

21. Bardin L. Análise de conteúdo. São Paulo: Edições 70; 2016.

22. Brasil. Conselho Federal de Nutricionistas B. Resolução CFN no 380 de 28 de dezembro de 2005. Dispõe sobre a definição das áreas de atuação do nutricionista e suas atribuições, estabelecer parâmetros numéricos de referência, por área de atuação e dá outras providências. [Internet]. Diário Oficial da União. 10 jan. 2006; 1:66-71. Retificada Diário Oficial da União. 10 ago. 2006; 1:52.

23. Pádua JG, Boog MCF. Avaliação da inserção do nutricionista na Rede Básica de Saúde dos municípios da Região Metropolitana de Campinas. Rev Nutr. 2006; 19(4):413-424.

24. Laporte-Pinfildi AS, Zangirolani LTO, Spina N, Martins PA, Medeiros MAT. Atenção nutricional no pré-natal e no puerpério: percepção dos gestores da Atenção Básica à Saúde. Rev Nutr. 2016; 29(1):109-123.

25. Cunha GT, Campos GWS. Apoio matricial e Atenção Primária em Saúde. Saúde Soc. 2011; 20(4):961-970.

26. Matumoto S, Fortuna CM, Mishima SM, Pereira MJB, Domingos NAM. Supervisão de equipes no Programa de Saúde da Família: reflexões acerca do desafio da produção de cuidados. Interface Comun Saúde, Educ. 2005; 9(16):9-24.

27. Medeiros MAT, Braga-Campos FC, Moreira MIB. A integralidade como eixo da formação em proposta interdisciplinar: estágios de Nutrição e Psicologia no campo da Saúde Coletiva. Rev Nutr. 2014; 27(6):785-798.

28. Albuquerque ABB, Bosi MLM. Visita domiciliar no âmbito da Estratégia Saúde da Família: percepções de usuários no Município de Fortaleza, Ceará, Brasil. Cad Saúde Pública. 2009; 25(5):1103-1112.

29. Demétrio F, Paiva JB, Fróes AAG, Freitas MCS, Santos LAS. A nutrição clínica ampliada e a humanização da relação nutricionista-paciente: contribuições para reflexão. Rev Nutr. 2011; 24(5):743-763.

30. Besen CB, Souza Netto M, Da Ros MA, Silva FW, Silva CG, Pires MF. A estratégia saúde da família como objeto de educação em saúde. Saúde Soc. 2007; 16(1):57-68.

31. Campos GWS, Amaral MA. A clínica ampliada e compartilhada, a gestão democrática e redes de atenção como referênciais teórico-operacionais para a reforma do hospital. Cienc Saúde Coletiva. 2007; 12(4):849-859.

32. Gomes MCPA, Pinheiro R. Acolhimento e vínculo: práticas de integralidade na gestão do cuidado em saúde em grandes centros urbanos. Interface - Comun Saúde, Educ. 2005; 9(17):287-301.

33. Tesser CD, Norman AH. Repensando o acesso ao cuidado na Estratégia Saúde da Família. Saúde Soc. 2014; 23(3):869-883.

34. Santos LAS. O fazer educação alimentar e nutricional: algumas contribuições para reflexão. Cienc Saúde Coletiva. 2012; 17(2):455-462. 
35. Aguiar CB, Costa NMSC. Formação e atuação de nutricionistas dos Núcleos de Apoio à Saúde da Família. Rev Nutr. 2015; 28(2):207-216.

36. Cervato-Mancuso AM. Elaboração de programas de educação alimentar e nutricional. In: Diez-Garcia RW, Cervato-Mancuso AM, editor. Mudanças alimentares e educação alimentar e nutricional. Rio de Janeiro: Guanabara-Koogan; 2011. p. 187-197.

37. Capozzolo AA, Imbrizi JM, Liberman F, Mendes R. Experiência, produção de conhecimento e formação em saúde. Interface - Comun Saúde, Educ. Comunicação, Saúde, Educação. 2013; 17(45):357-370.

38. Viana MR, Neves, A S, Camargo Junior KR, Prado SD, Mendonça, ALO. A racionalidade nutricional e sua influência na medicalização da comida no Brasil. Ciênc Saúde Coletiva. 2017; 22(2):447-456.

Recebido: 27 de outubro, 2017

Revisado: 06 de fevereiro, 2018

Aceito: 27 de fevereiro, 2018 